\title{
Electrolysis of Alcohols in High Temperature-High Pressure Water
}

\author{
Asli Yuksel ${ }^{1}$, Mitsuru Sasaki², Motonobu Goto ${ }^{3}$ \\ ${ }^{1}$ Chemical Engineering, Izmir Institute of Technology, Izmir, TURKEY \\ ${ }^{2}$ Applied Chemistry and Biochemistry, Kumamoto University, Kumamoto, JAPAN \\ ${ }^{3}$ Chemical Engineering, Nagoya University, Nagoya, JAPAN
}

\begin{abstract}
The design of clean, efficient and environmentally friendly routes that reduce the waste production and fuel emissions of pollutants into the atmosphere, produce clean, affordable, and renewable energy sources to lessen energy consumption and toxicity on the environment, has become a central issue of chemical research both in industry and academia. One of the approaches being used in green chemistry practices is to use water as a solvent and reaction medium where possible. Much of this work deals with liquid water at temperatures exceeding the normal boiling point which is denoted as sub-critical water.

Electrochemical reaction, usually operated at atmospheric condition in water, is generally slow, although it has advantages over chemical reaction such as suppression of side reaction by tuning operating conditions. Since sub-critical water $\left(7 \mathrm{MPa}\right.$ and $\left.250{ }^{\circ} \mathrm{C}\right)$ has remarkable properties such as high ion product and low dielectric constant, it could be a suitable reaction media. We have been studying electrolysis of organic compounds in sub-critical water as waste treatment and molecular degradation technologies. Electrolysis in sub-critical water could degrade harmful and thermally stable organic compounds into innocuous compounds such as hydrogen and water. In this research, we focused on the investigation of the electrochemical reactions of alcohols in sub-critical water to evaluate possibility for the selective production of hydrogen and value-added chemicals.

Electrochemical reactions were carried out in sub-critical water using a specially designed autoclave made of SS 316 with a volume of $500 \mathrm{~mL}$. For comparison, thermal degradation experiments of alcohols were also conducted without any direct current loading at identical conditions. Here we employed glycerol and 1-butanol as model compounds of alcohols. As a result of 1-butanol experiments, butanal and butyric acid were produced via partial oxidation at $250{ }^{\circ} \mathrm{C}$ and by applying 1-3 A of direct current while no oxidation products were observed at the hydrothermal degradation run. As a gaseous product, hydrogen gas was generated according to the electrochemical reaction mechanism. In the case of glycerol experiments, the main gaseous product was hydrogen gas, whereas glycolaldehyde, lactic acid, and formic acid were generated as the main liquid products at $280{ }^{\circ} \mathrm{C}$. Results indicated that greater than $92 \%$ of the glycerol could be decomposed under optimum conditions by hydrothermal electrolysis technique.

This presented research will help to degrade stable organic materials in an environmentally friendly way and without need for secondary treatment processes. It will also address the need for novel more efficient techniques for the degradation of stable organic compounds in aqueous conditions and it will advance the use of water as a reaction medium in an efficient way without any organic solvent.
\end{abstract}

Keywords: Sub-critical water, hydrothermal electrolysis, glycerol, 1-butanol 


\section{INTRODUCTION}

Nowadays, biodiesel fuels have garnered much attention because they have various advantages as alternative to petroleum-based fuel, e.g., they are renewable, non-toxic, possess a favorable energy balance and have lower harmful emissions. However, this rise in the production of biodiesel has created the problem of disposing of the glycerol that is generated as the major by-product. Presently, in many biodiesel plants with low capacity, this crude glycerol is discharged in the wastewater together with some methanol, alkali and low concentration of fatty acids. As a result of this, both environmental pollution and economical losses are generated. The conversion of glycerol to various compounds has been investigated recently. Catalytic dehydration of glycerol in sub- and supercritical water and its reaction mechanism have been studied. It was found that the $\mathrm{OH}$ group on various catalysts can catalyze the dehydration of glycerol to 3-hydroxypropionaldehyde, 1,3-propanediol, acrolein and other degradation products through subsequent hydrogenation [1-3]. In another study, ionic reactions and pyrolysis of glycerol as competing reaction pathways in near- and supercritical water have been investigated $[4,5]$. Moreover, pyrolysis of glycerol has been studied in steam with the major products of acrolein, formaldehyde and acetaldehyde [6, 7]. At higher temperatures, other products such as carbon dioxide, molecular hydrogen, ethylene and methane were observed, as an indication of more complex chemistry.

One method of particular interest is the conversion of glycerol to lactic acid. Kishida et al. [8] showed that glycerol can be converted into lactic acid by hydrothermal decomposition experiments of glycerol in sub-critical water under alkaline conditions. Studies on the conversion pathway have suggested that glycerol was first decomposed to pyruvaldehyde with elimination of hydrogen by a $\mathrm{H}$ - shift to the adjacent hydrogen. The pyruvaldehyde was then converted into lactic acid ion by the benzilic acid rearrangement. In addition to lactic acid, small amounts of pyruvaldehyde, acetic acid and formic acid were detected.

The main purpose of this work is to evaluate the electrolytic reaction of alcohols (glycerol and 1butanol) under hydrothermal conditions to create a new technology in an environmentally friendly manner. We then compared the results obtained by this process with wet electrolytic oxidation (WEO) from the viewpoints of reaction- controlling parameters, possible reaction scheme, current efficiency and oxidation characteristics of alcohols.

In this study, sub-critical water was selected as a reaction medium because it is cheap, non-toxic, non-flammable, non-explosive and offers essential advantages compared to other substances, particularly in the field of "green chemistry" [9]. Recent studies have demonstrated that heating water above its boiling point under enough pressure to maintain its liquid state causes some physicochemical changes that are useful in reducing the rate problems as well as increasing the solubility of organics in water. For example, the dielectric constant is much lower, and the number of hydrogen bonds is diminished. Additionally, the ion product or dissociation constant $\left(\mathrm{K}_{\mathrm{w}}\right)$ for water as it approaches the critical point is about three orders of magnitude higher than it is for ambient liquid water. As a result, water becomes an excellent solvent for organic compounds. It can also boast a higher $\mathrm{H}^{+}$and $\mathrm{OH}^{-}$ion concentrations than liquid water under certain conditions $[10,11]$. In the case of economic feasibility, sub-critical water minimizes the amount of energy consumed for electrolysis. Because the "current efficiency" as a function of the reaction progress increases with increasing the temperature of water toward its critical point $[12,13]$. 


\section{EXPERIMENT}

1-Butanol (99.0\%) and glycerol (99.0\%), obtained from Wako Pure Chemicals Industries Ltd. were used as starting materials. Other chemicals were also purchased from the same company and they were used without further purification. The experiments of hydrothermal electrolysis were carried out in batch reactor at various reaction temperatures $\left(200-250{ }^{\circ} \mathrm{C}\right)$. This autoclave, illustrated in Figure 1, made of stainless steel with a volume of $500 \mathrm{~mL}$. In this reactor configuration, a cylindrical electrode made of iridium was used as an anode and the titanium beaker was used as a cathode to prevent corrosion.

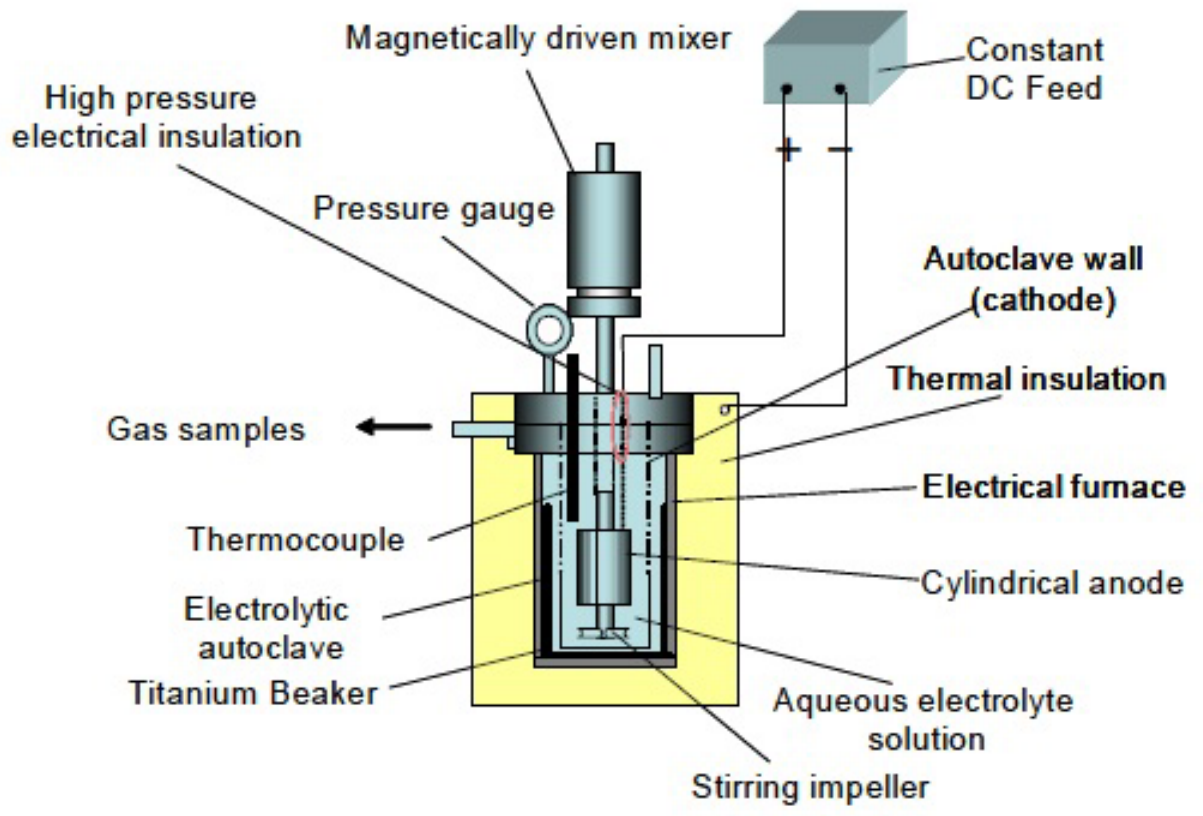

Figure 1. Hydrothermal electrolysis autoclave.

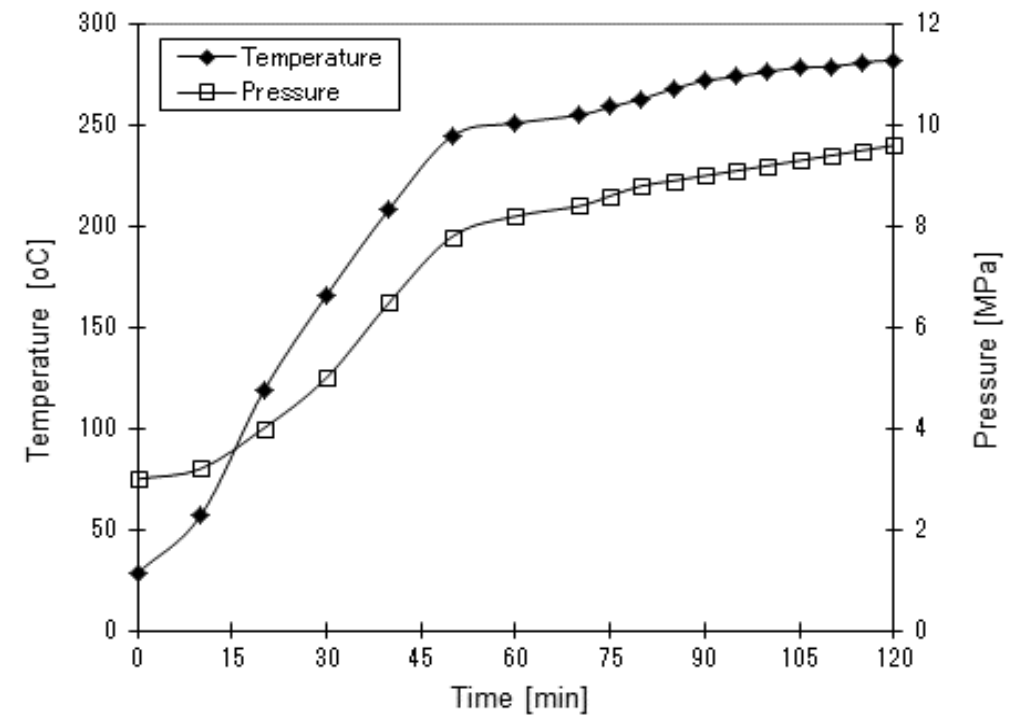

Figure 2. Typical pressure-temperature profile for hydrothermal electrolysis at $280{ }^{\circ} \mathrm{C}$ with an initial pressure of $3 \mathrm{MPa}$. 
At the beginning of the experiment, the aqueous solution $(250 \mathrm{~mL})$ was loaded in titanium beaker and placed in the autoclave. After its lids were closed tightly, the air in the autoclave was purged and pressurized with argon gas. This internal pressure of the autoclave was defined as the initial pressure of the experiment. The profile of internal pressure in the autoclave is plotted in Figure 2. The electric current was applied in the autoclave when the whole system reached the desired condition. In the hydrothermal degradation experiments, no current was passed through the electrodes.

Both gas and liquid samples were collected and analyzed at the end of the reaction. Solid samples were not specifically analyzed, because no measurable solid products were observed. Gas formed during the reaction was collected in gas sampling plastic bags and analyzed by gas chromatography with a thermal conductivity detector (GC-TCD). Liquid products were identified by gas chromatography-mass spectrometry (GC-MS) and quantified by gas chromatography with a flame ionization detector (GC-FID) and high performance liquid chromatography (HPLC).

\section{RESULTS AND DISCUSSION}

Figure 3 illustrates the GC-MS chromatogram of the compound produced from 0.27 $\mathrm{mol} / \mathrm{L}$ of 1 -butanol reaction at $250{ }^{\circ} \mathrm{C}$ and $8 \mathrm{MPa}$ after $60 \mathrm{~min}$ of reaction time with and without dc current. The GC-MS traces exhibit a number of small features indicating the presence of lower and higher molecular weight products that varied as a function of temperature. The products were identified by matching with the available mass spectra data in the GC-MS computer library. Figure 3 shows that 2-butene, 5-methyl-3-hexanol and butyl ether were detected clearly as products of the decomposition of 1-butanol when no current was applied. In sub-critical region, the ion product of water is higher than it is for liquid ambient water. As a result of this change in the thermodynamic property of water as it approaches the critical point, reactions are generally catalyzed by protons or hydroxyl ions produced in the solution without the external addition of acids or bases. At hydrothermal electrolysis, the decomposition of 1butanol produced butanal and butyric acid as main products. $\mathrm{KCl}$ which was added as an electrolyte was dissolved completely in water at the reaction temperature of 200 and $250{ }^{\circ} \mathrm{C}$. This compound was considered as an inert electrolyte that would enhance the ionic strength of the aqueous reaction medium and hence would influence the rate of ionic reaction, according the Bronsted-Bjerrum effect for ionic reactions in solution. However, the most important step is the protonation of 1-butanol. This means that the reaction rates of the ion reactions not only depend on the existence of $\mathrm{KCl}$ as an electrolyte solution but also depend on the self-dissociation of water. Solution of $\mathrm{KCl}$ in liquid water prevails as separate $\mathrm{K}^{+}$and $\mathrm{Cl}^{-}$ions. Due to charge interactions these distribution generate dipole properties within the water molecule. On the other hand, the electron transfer from anode surface also promotes the oxidation of 1-butanol. In this part, butanal and butyric acid were quantified, since these compounds were considered to be the main products of the hydrothermal electrolysis of 1-butanol.

In the case of the hydrothermal electrolysis of glycerol with alkali, lactic acid (L- and D-) and hydrogen gas were formed as main organic acid and a gaseous product, respectively. Additionally, small amounts of formic acid, glycolic acid and aldehydes were produced. Figure 4 shows the glycerol conversion under various operating conditions by batch reactor. 


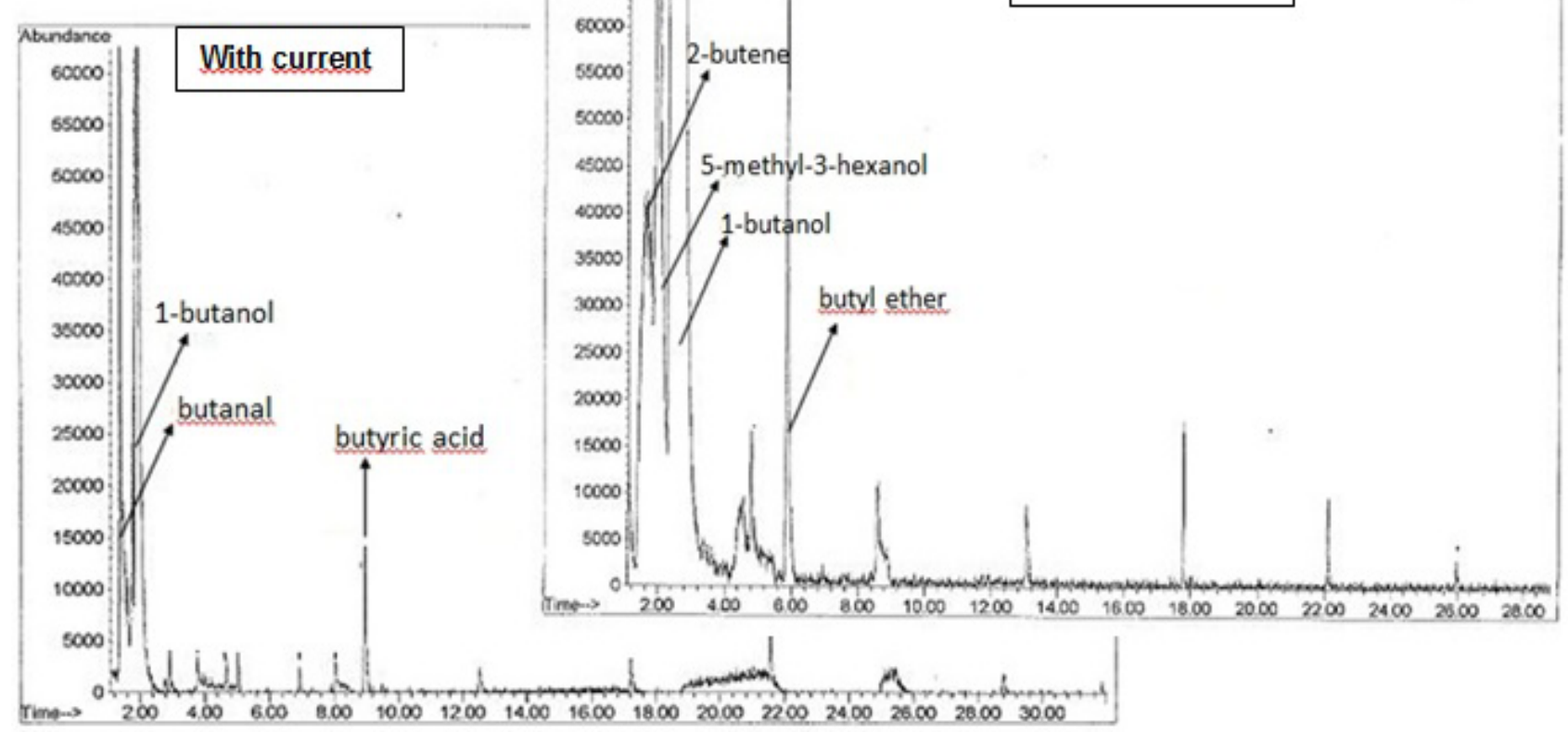

Figure 3. GC-MS chromatogram of the liquid phase recovered after treatment of 1-Butanol at $250{ }^{\circ} \mathrm{C}$ with and without de current.

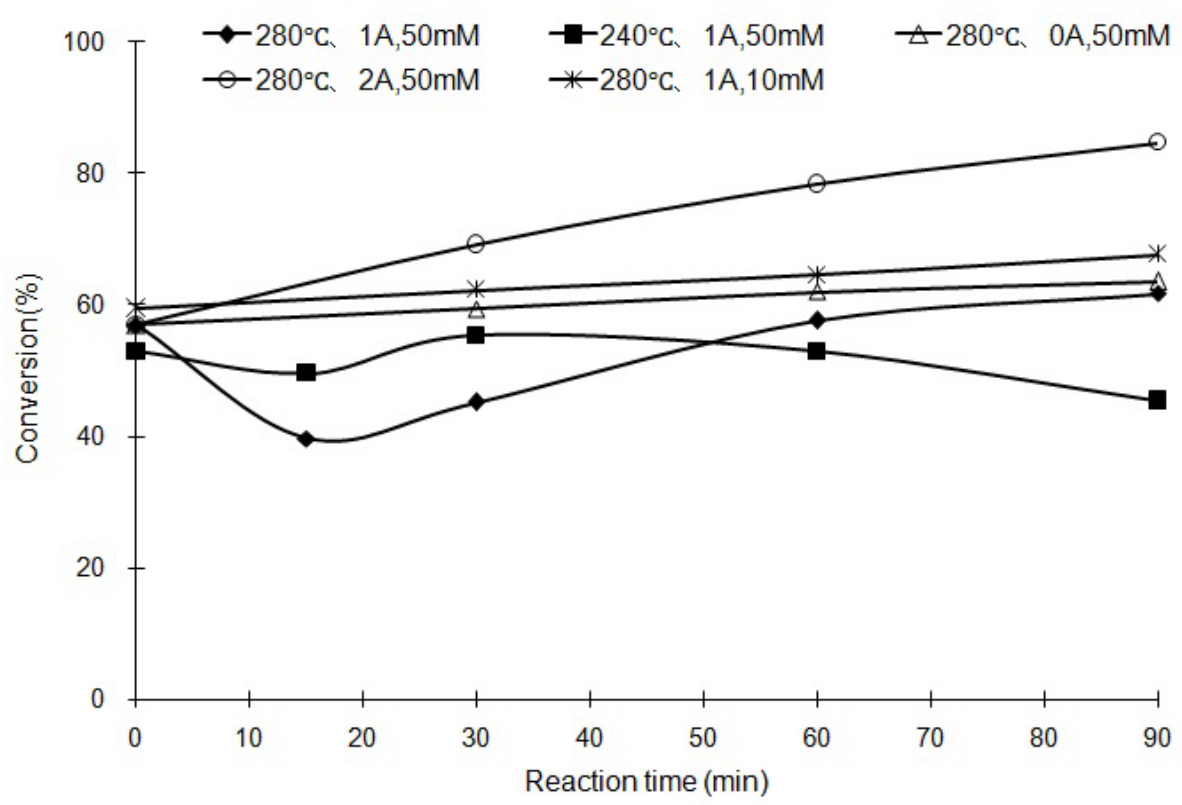

Figure 4. Conversion of glycerol under various operating conditions by batch reactor.

Conversion of glycerol under alkaline conditions increased with increasing electrolysis current. When 2 A current was applied during $90 \mathrm{~min}$, it reached its maximum value as $84 \%$ at $280{ }^{\circ} \mathrm{C}$. As for $\mathrm{NaOH}$ effect, by increasing the concentration of alkali from 10 to $50 \mathrm{mM}$ only $6 \%$ difference in the conversion value was observed at the end of $90 \mathrm{~min}$. When the applied current was raised from $0 \mathrm{~A}$ to $1 \mathrm{~A}$, the conversion of glycerol was almost same with hydrothermal 
degradation run (0 A). Although current was applied during $90 \mathrm{~min}$, there was still unreacted glycerol. There may be several seasons: one of them is dead volume. The autoclave includes two valves and pressure gauge which may include some dead volumes. Another possibility may be related to the concentration of alkali. In our system, we tried to keep $\mathrm{NaOH}$ concentration as low as possible (at most $50 \mathrm{mM}$ ) in order to decrease the risk of corrosion which is higher in the electrolytic systems.

As main high-value product, lactic acid was produced from the hydrothermal electrolysis of glycerol under alkaline conditions by using autoclave. At $280{ }^{\circ} \mathrm{C}$, with $50 \mathrm{mM} \mathrm{NaOH}$ concentration and by applying 1 A current, maximum lactic acid yield was recorded as $35 \%$ after 90 min electrolysis time.

\section{CONCLUSIONS}

Hydrothermal electrolysis experiments using aqueous 1-butanol and glycerol solutions as model alcohols were carried out under a wide range of operating conditions using a specially designed autoclave. Despite the uncertainties remaining concerning the oxidation mechanism, differences in the governing reactions of hydrothermal electrolysis were clearly revealed. The experiments showed that although 1-butanol and glycerol are very stable compound under high-temperature and high-pressure aqueous conditions, hydrothermal electrolysis has an enhancing effect on the decomposition of these materials. The fundamental reaction behavior of 1-butanol and glycerol as model alcohols was analyzed under a wide range of operating conditions. Main liquid products from the hydrothermal electrolysis of 1-butanol with $\mathrm{KCl}$ in sub-critical water were butanal and butyric acid, whereas in the case of glycerol degradation with an alkali, formic acid, glycolic acid and lactic acid were formed as main organic acids. It was found that the conversion of 1-butanol and glycerol increased with the increasing reaction time and applied current value.

\section{REFERENCES}

1. L. Ott, M. Bicker and H. Vogel, Green Chem.8, 214 (2006).

2. V. Lehr, M. Sarlea, L. Ott and H. Vogel, Catalysis Today 121, 121 (2007).

3. M. Watanabe, T. Lida, Y. Aizawa, T. M. Aida and H. Inomata, Bioresource Technology 98, 1285 (2007).

4. W. Buhler, E. Dinjus, H. J. Ederer, A. Kruse and C. Mas, Journal of Supercritical Fluids 22, 37 (2002).

5. T. Valliyappan, N. N. Bakhsi and A. K. Dalai, Bioresource Technology 99, 4476 (2008).

6. M. J. Antal, W. S. L. Mok, J. C. Roy, A. T. Raissi and D. G. M. Anderson, J. Anal. Appl. Pyrolysis 8,291 (1985).

7. Y. S. Stein, M. J. Antal and M. J. Jones, J. Anal. Appl. Pyrolysis 4, 283 (1983).

8. H. Kishida, F. Jin, Z. Zhou, T. Moriya and H. Enomoto, Chemistry Letters 34, 11 (2005).

9. T. Rogalinski, K. Liu, T. Albrecht and G. Brunner, J. of Supercritical Fluids 46, 335 (2008).

10. T. Clifford, Fundamentals of Supercritical Fluids (Oxford University Press, New York, 1998) p. 23.

11. P. E. Savage, Chem. Rev. 99, 603 (1999).

12. F. S. Asghari and H. Yoshida, J. Phys. Chem. A 112, 7402 (2008).

13. A. Yuksel, H. Koga, M. Sasaki and M. Goto, J. Renewable Sustainable Energy 1, 033112 (2009). 